\title{
A giant benign epicardial lipomatosis of the left ventricle
}

\author{
Trushar Gajjar, Nageswar Rao, Neelam Desai \\ Sri Sathya Sai Institute of Higher Medical Sciences - Prasanthigram, CTVS Department, Prasanthigram, Andhra Pradesh, India
}

Received: August 15, 2016 Accepted: August 30, 2016 Published online: January 11, 2017

\begin{abstract}
ABSTR ACT
Fatty masses of the heart are relatively uncommon. Herein, we report an extremely rare case of a giant benign epicardial lipomatosis arising from the left ventricle. A 26-year-old female presented with atypical chest pain and easy fatigability for the past six months. Chest X-ray showed an increased cardiothoracic ratio. Echocardiography revealed a large homogenous mass in the pericardial space near the lateral surface of the left ventricle. Computed tomography and magnetic resonance imaging of the chest showed a large fat-density mass with well-defined margins, arising from the left ventricle. The patient underwent surgical removal of the mass without cardiopulmonary bypass. She recovered well and discharged in the seventh postoperative day.
\end{abstract}

Keywords: Benign cardiac tumor; cardiac malignancy; epicardial lipomatosis; left ventricle.

Primary cardiac tumors account for 5 to $10 \%$ of all neoplasms of the heart and pericardium with an incidence ranging from 0.0001 to $0.05 \%$ in autopsies. ${ }^{[1]}$ Lipomas are the second most common benign cardiac tumors. ${ }^{[2]}$ These tumors are often asymptomatic in nature and usually detected incidentally, mostly during autopsies. ${ }^{[1-3]}$ Although a lipoma is a benign tumor, it can lead to compression of cardiac chambers and cause life-threatening complications, when the tumor size increases. ${ }^{[1-6]}$ Herein, we report a rare case of a giant benign epicardial lipomatosis arising from the left ventricle.

\section{CASE REPORT}

A 26-year-old female had atypical chest pain and easy fatigability for the past six months. Chest $\mathrm{X}$-ray showed a cardiothoracic ratio of $70 \%$. Echocardiography (ECHO) showed a large homogenous, echogenic mass in the pericardial space located along the lateral surface of the heart, arising from the epicardium and extending posterior, anterolateral surfaces of the left ventricle (LV) including the apex with minimal pericardial effusion (Figure 1a). The biventricular function was good with no regional wall motion abnormalities. Computed tomography (CT) of the chest showed a large fat-density mass, extending from the arch of the aorta superiorly to the dome of the diaphragm inferiorly in the left hemithorax. The mass had a well-defined lateral margin limited by the pericardium peripherally and its medial border was encasing the left atrium, LV and a part of right ventricle $(\mathrm{RV})$. The mass also encased the left anterior descending artery and derived its blood supply from the branches of the left coronary artery (Figure 1b). The tumor was found to be causing a mass effect on the left lower lobe of the lung and its vasculature. Cardiac magnetic resonance imaging (MRI) showed an abnormal soft tissue enhancement in the anterolateral surface of the heart occupying the anterolateral, posterior and inferior surfaces of the LV and anteriorly extending over the RV (Figure 1c). The mass was well-defined and had the same signal intensity as subcutaneous fat, in all the images and occupying almost the whole of the left hemithorax (Figure 1d). The mass was in close contact with the cardiac chambers, but not compressing any chambers or great vessels.

\section{Surgical operation}

The patient was taken up for surgery via median sternotomy without cardiopulmonary bypass (CPB). During opening the pericardium, a very large yellowish tumor with a smooth surface was detected covering the

Corresponding author: Trushar Gajjar, MD. Sri Sathya Sai Institute of Higher Medical Sciences - Prasanthigram, CTVS Department, Prasanthigram, 515134 Andhra Pradesh, India.

Tel: +91 9700355411 e-mail: trushargajjar@gmail.com 

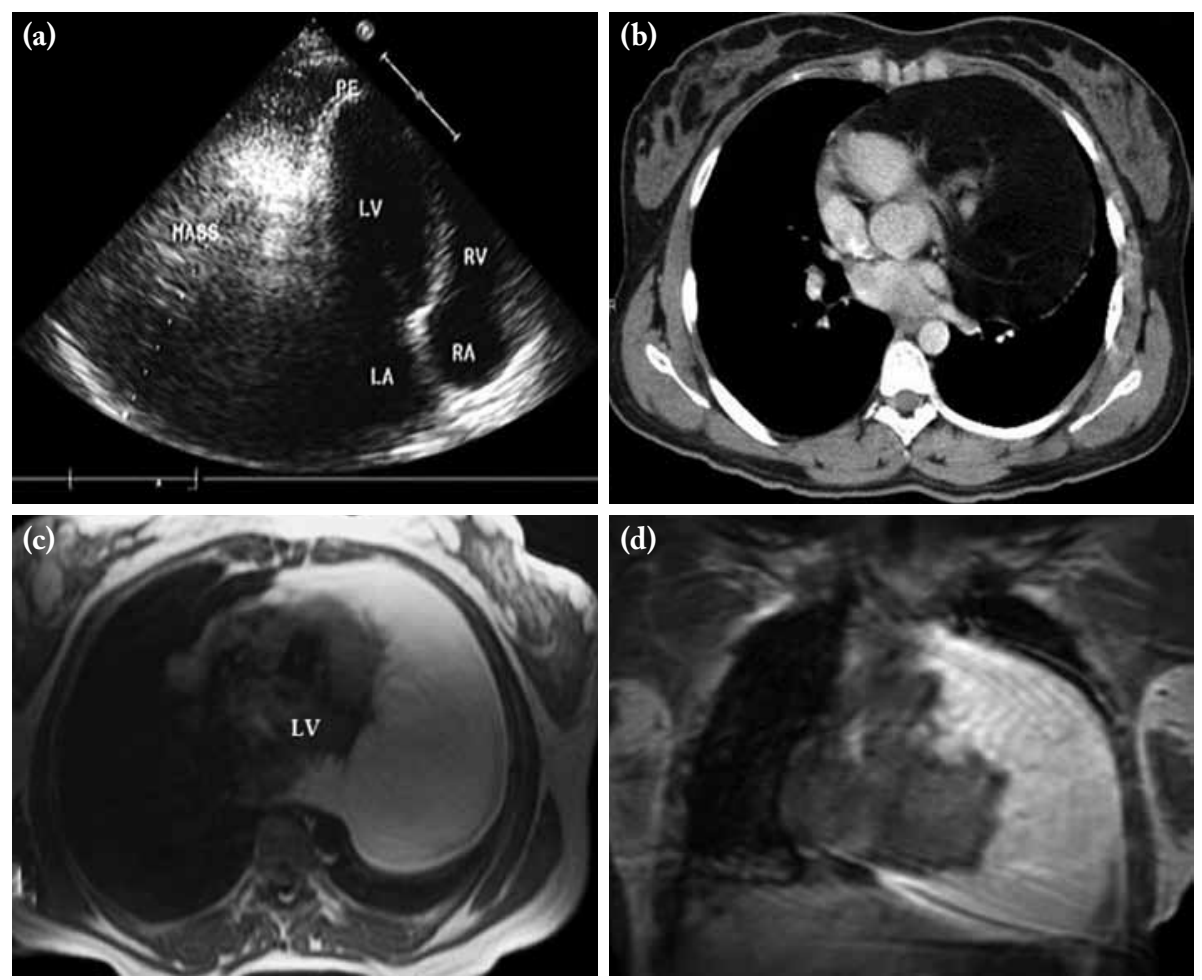

Figure 1. (a) Echocardiography showing a homogenous echogenic mass in the pericardial space along the lateral surface of the heart. (b) A computed tomography scan showing a large fat density mass with major vessels. (c) A magnetic resonance imaging scan showing a large fatty mass arising from the anterolateral and posterior surfaces of the left ventricle. (d) A magnetic resonance imaging scan showing the mass occupying almost the whole left hemithorax.

LV: Left ventricle; RV: Right ventricle; RA: Right atrium; LA: Left atrium; PE: Pericardial effusion.

entire heart and bulging into the wound, which was unable to be easily lifted out of the pericardial sac (Figure 2a). It had no definite stalk or pedicle and was firmly adherent to the LV. The tumor was highly vascular and received its blood supply from the left anterior descending artery and lots of feeding vessels arose directly from the epicardium of the LV. Major vascular branches supplying the tumor were ligated and clipped (Figure 2b). While the major portion of the tumor was excised, a thin rim of the tissue was left behind (Figure 2c), due to its firm adherence to the LV myocardium \& extreme vascularity. The excised specimen weighed around $850 \mathrm{~g}$. The postoperative course was uneventful and the patient was discharged in the seventh postoperative day. Histopathological examination showed the presence of fibroadipose tissues which revealed mature adipocytes arranged in lobules without cellular atypia, suggestive of lipomatosis (Figure 2d).

\section{DISCUSSION}

Benign tumors of the heart were rare. Lipomas are the second most common benign cardiac tumors. ${ }^{[2]}$ Cardiac lipomas represent about $10 \%$ of all cardiac tumors and are thought to originate either from the subendocardium, subpericardium or the myocardium. ${ }^{[3]}$ The majority of the cardiac lipomas are subendocardial or epicardial, and only $25 \%$ are found in the myocardium. ${ }^{[1-4]}$ Clinical presentation usually depends on the size and location of the tumor. They usually originate from the epicardial fat tissue and grow slowly into the pericardial sac; in case of asymptomatic cases, they often achieve enormous dimensions before producing any symptoms. ${ }^{[1-4]}$ Many tumors are clinically silent, being found only during necropsy or on routine chest X-rays. ${ }^{[1-4]}$ These tumors cause symptoms through compression of the heart. Anginal pain due to coronary arterial compression, atrial arrhythmias, 

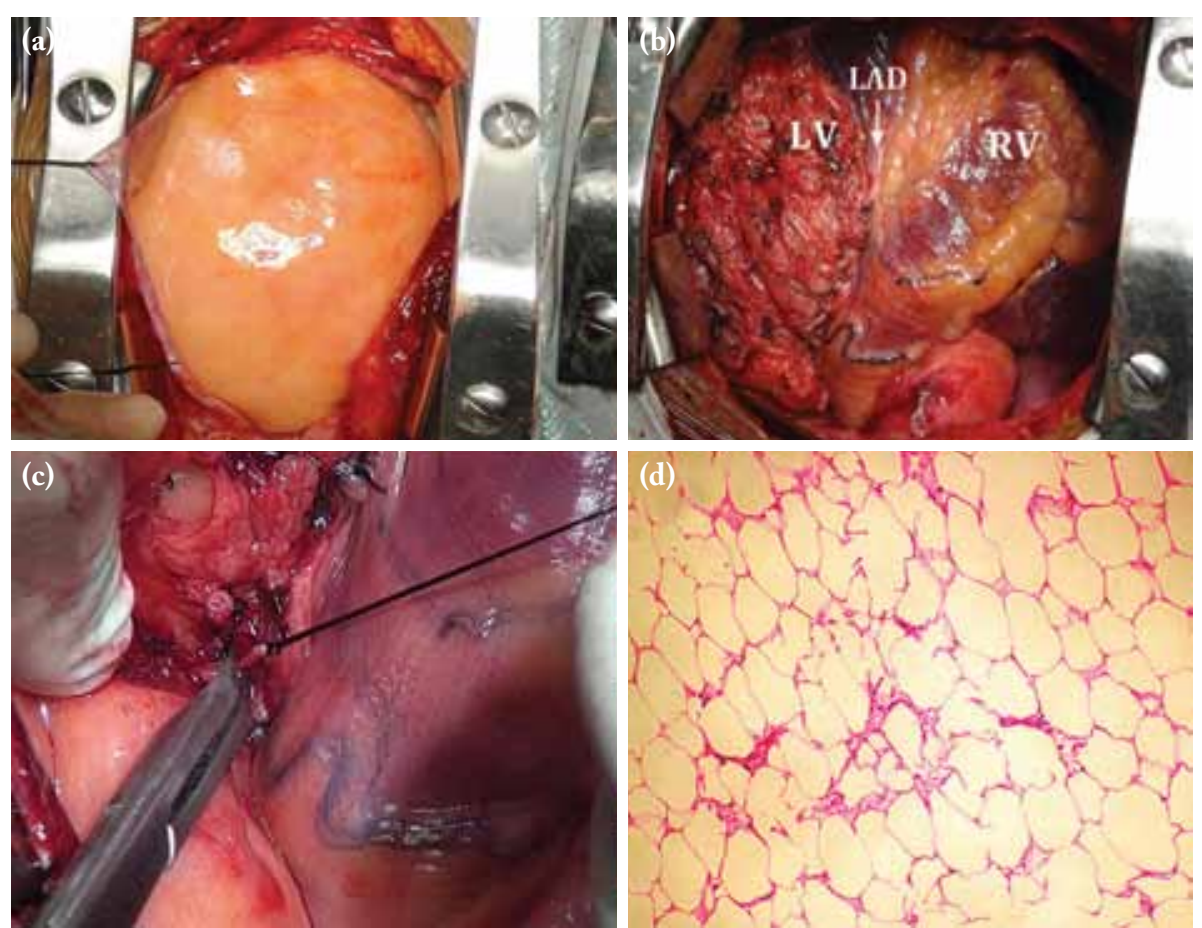

Figure 2. (a) An intraoperative view showing the mass just after opening the pericardium. (b) Major vessels supplying the tumor which were ligated and clipped. (c) Showing the left ventricle after excision of the major portion of the tumor and normal right ventricle. (d) Histopathological examination with a paraffin section of lipomatosis showing mature adipocytes (H-E x 400).

LAD: Left anterior descending artery; LV: Left ventricle; RV: Right ventricle.

and effort intolerance has been also reported. ${ }^{[4]}$ The subendocardial tumors with an intracavitary extension may cause symptoms characteristic of their location; they usually produce early symptoms, such as congestive heart failure, supraventricular or ventricular arrhythmias, syncope, or sudden cardiac death. ${ }^{[5]}$ Chest X-ray usually does not differentiate between pericardial effusion and a subepicardial lipoma. In addition, ECHO accurately defines the location and extent of the mass. ${ }^{[1-4]}$ However, the acoustic appearance of an intra-pericardial lipoma is not diagnostic. Subepicardial lipomas usually appear relatively ECHO-lucent and may be erroneously interpreted as the pericardial fluid. ${ }^{[4]}$ Also, CT and MRI can demonstrate lipomatous tumors. ${ }^{[1-4]}$ The latter can distinguish between lipomas and other tumors, such as liposarcomas. It also provides a clear definition of blood vessels to precisely detect the location, size, and extension of the tumor. ${ }^{[1-4]}$ It also demonstrates the relationship of the tumor to the coronary arteries ${ }^{[1]}$ and estimates the intramyocardial extension. These are important factors in determining tumor resectability and help planning the management approach. ${ }^{[4]}$ Although some epicardial tumors may be removed without $\mathrm{CPB}$, most intramural and intracavitary tumors should be excised with the use of $\mathrm{CPB}$, as cardiac manipulation may release part of the tumor, causing an embolism. Following the first successful removal of an intrapericardial lipoma by Maurer in 1952, several sporadic cases have been reported. ${ }^{[1-6]}$ Benign lipomas can be mostly excised completely with low morbidity and mortality and with excellent long-term results. ${ }^{[1]}$ In the majority of cases, intrapericardial lipomas may be removed without CPB. However, $\mathrm{CPB}$ may be required in cases of recurrence and when there is an intracardiac extension. The decision to resect such epicardial lipomatosis is going to be debulking, as it is densely adherent to the heart and major coronary arteries. Off pump debulking allows ease of identifying bleeders which is going to be a much more difficult job on the pump, since the heart is arrested and bleeders would not be seen clearly. Intraoperatively, it is important to excise the entire 
tumor along with the pedicle to prevent recurrences. The rate of lipoma recurrence after total and subtotal resection is very low. ${ }^{[6]}$ Our case seems to be exceptional, as this was a giant benign lipomatous tumor without any pedicle directly arising from the LV surface. To the best of our knowledge, this is the first case reported in the literature.

In conclusion, most of the patients with intrapericardial lipomas are asymptomatic for a long time due to the slow growth of the tumor. Imaging modalities such as computed tomography and magnetic resonance imaging are the main diagnostic tools. Surgical resection is necessary to prevent tumor compression syndromes of the heart. Finally, the status of malignancy needs to ruled out and confirmed by histological examination.

\section{Declaration of conflicting interests}

The authors declared no conflicts of interest with respect to the authorship and/or publication of this article.

\section{Funding}

The authors received no financial support for the research and/or authorship of this article.

\section{REFERENCES}

1. Miralles A, Bracamonte L, Soncul H, Diaz del Castillo R, Akhtar R, Bors V, Pavie A, et al. Cardiac tumors: clinical experience and surgical results in 74 patients. Ann Thorac Surg 1991;52:886-95.

2. Burke A, Virmani R. Tumors of the heart and great vessels. In: Atlas of tumor pathology. Washington: Armed Forces Institute of Pathology; 1996.

3. Hananouchi GI, Goff WB 2nd. Cardiac lipoma: six-year follow-up with MRI characteristics, and a review of the literature. Magn Reson Imaging 1990;8:825-8.

4. Doshi S, Halim M, Singh H, Patel R. Massive intrapericardial lipoma, a rare cause of breathlessness. Investigations and management. Int J Cardiol 1998;66:211-5.

5. Reynen K, Rein J, Wittekind C, von der Emde J. Surgical removal of a lipoma of the heart. Int J Cardiol 1993;40:67-8.

6. Bardakci H, Altintas G, Unal U, Kervan U, Arda K, Birincioglu L. Giant cardiac lipoma: report of a case. J Card Surg 2008;23:254-6. 